\title{
Renormalization Scheme for Self-Organized Criticality in Sandpile Models
}

\author{
L. Pietronero, A. Vespignani, and S. Zapperi \\ Dipartimento di Fisica, Università di Roma "La Sapienza" Piazzale A. Moro 2, 00185 Roma, Italy
}

(Received 1 October 1993)

\begin{abstract}
We introduce a renormalization scheme of novel type that allows us to characterize the critical state and the scale invariant dynamics in sandpile models. The attractive fixed point clarifies the nature of self-organization in these systems. Universality classes can be identified and the critical exponents can be computed analytically. We obtain $\tau=1.253$ for the avalanche exponent and $z=1.234$ for the dynamical exponent. These results are in good agreement with computer simulations. The method can be naturally extended to other problems with nonequilibrium stationary states.
\end{abstract}

PACS numbers: $64.60 . A k, 02.50 .-r, 05.40 .+\mathrm{j}$

Sandpile models, introduced by Bak and co-workers [1], represent an interesting case of dynamically driven systems that evolve spontaneously in a stationary critical state. This is an example of self-organized criticality (SOC) in which a system with many interacting degrees of freedom and with short range couplings self-stabilizes in a marginally stable state in which a disturbance can lead to avalanches of all sizes. The distribution of avalanches is characterized by a power law $P(s)=s^{-\tau}$ in which $s$ is the number of sites involved in a relaxation process. A second exponent $(z)$ characterizes the dynamics by linking time and linear extension in a single avalanche: $t=l^{z}$. With respect to usual statistical mechanics problems these systems are characterized by a nonlinear dynamics and by the fact that the critical state is reached spontaneously without the fine tuning of any critical parameter. Fractal growth phenomena [2], like diffusion limited aggregation (DLA), are another example of SOC, in the sense that they lead spontaneously to complex fractal patterns. Various authors [3] believe, however, that both DLA and the sandpile models pose questions of a new type for which it would be desirable to define a common theoretical scheme. The attempts to apply usual renormalization schemes to these models have been problematic because self-criticality implies the absence of "relevant" critical parameters from which usual scaling can be defined. For DLA-like problems we have introduced a new theoretical framework, the fixed scale transformation (FST) [4], that is based on two elements: One is the stability with respect to the dynamical evolution and the second is the identification of the scale invariant dynamics. This second element corresponds essentially to a renormalization scheme in which, however, the critical exponents are related directly to the fixed point properties of the dynamics, instead of their derivative with respect to the relevant critical parameter, that in these SOC problems does not exist.

In this Letter we follow the same reasoning for the sandpile model and develop a renormalization scheme for its dynamics. This permits us to clarify the SOC nature of the process, to identify the universality classes, and to compute analytically the critical exponents $\tau$ and $z$.

There are several sandpile models; the first class is the one introduced by Bak and co-workers [1] in which the instability is defined by the critical height. A second class of models is defined with respect to the critical slope and it appears to belong to a different universality class $[5,6]$. For these models there have been extensive studies by computer simulations [5-9] and some exact results have been derived by Dhar and co-workers [10] with group theoretical arguments. These models have also been generalized to the case in which the local conservation laws are violated to simulate dissipation $[11,12]$. This leads to a relevant modification of the SOC properties. In general these results are discussed in terms of scaling arguments $[1,5,13,14]$. SOC properties have also been identified in models related to invasion percolation for which a field theory approach has recently been proposed [15].

Our discussion will refer to the critical height models. These models are cellular automaton defined on a lattice. A variable (energy) is assigned to each site; energy is then added randomly on the system. When the energy of a site reaches a critical value, its entire energy is released to the neighboring sites in various ways. These may become unstable in their turn and so on. The system has open boundary conditions that allow the dissipation of energy outside. In full generality we can characterize in all these models three classes of sites (Fig. 1):

(i) White sites are those whose energy (height) is far from the threshold value. This implies that the addition of a "quantum" of energy will not induce relaxation.

(ii) Black sites are those whose energy is critical in the sense that the addition of a "quantum" of energy will introduce relaxation.

(iii) Encircled sites are unstable sites that will relax at the next time step according to the specific rules assigned. An example of this relaxation is shown in the lower part of Fig. 1. In this case the relaxation of the central site induces a redistribution of the energy over two of the neighbors, as in the model of Manna [8], and these become critical.

We should now extend the characterization of the static and dynamical properties of the system at a generic scale $b$, by considering coarse grained variables. A cell of size $b$ relaxes if subrelaxation processes span the cell and transfer energy to some neighbors. Independently of 


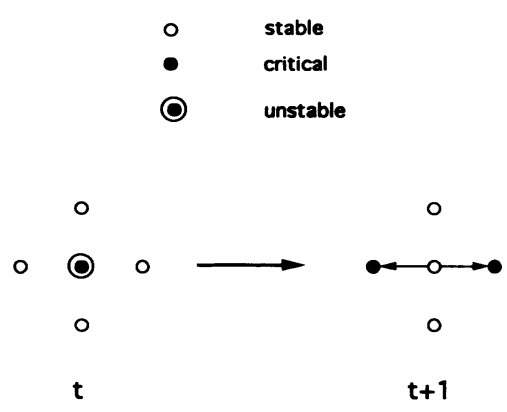

FIG. 1. Upper part: classification of the three possible classes of sites: stable (white) sites do not relax if a "quantum" of energy is added. Critical (black) sites would relax if energy were added. Unstable (encircled) sites will relax at the next time step. In the lower part we show an example of the relaxation process.

the minimal scale dynamics such a relaxation process, for a coarse grained cell of size $b$ in a square lattice, can lead to four possible situations as shown in Fig. 2. Energy can be transferred to one, two, three, or four neighbors with a probability distribution defined by the vector

$$
\bar{P} \equiv\left(p_{1}, p_{2}, p_{3}, p_{4}\right), \quad \sum_{n=1}^{4} p_{n}=1 .
$$

This vector characterizes the phase space for the relaxation dynamics at a generic scale. In this framework the model of Manna [8] distributes the energy to only two neighbors. This implies that its small scale dynamics is defined by the vector $\bar{P}^{(0)} \equiv(0,1,0,0)$. The model of Bak, Tang, and Wiesenfeld (BTW) [1] distributes the energy to all four neighbors and it corresponds to $\bar{P}(0)$ $\equiv(0,0,0,1)$. We are going to see that, under scale trans-

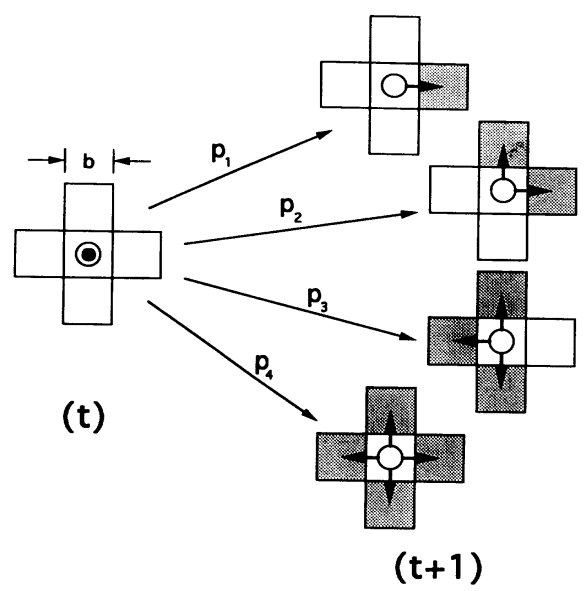

FIG. 2. No matter what is the small scale dynamics, at a generic scale $(b)$ relaxation can occur in the four possible ways shown. These four possibilities characterize the phase space of the dynamics of the system that will be used in the relaxation scheme. The probabilities refer to the number of neighboring cells influenced by the relaxation, independently of their position.
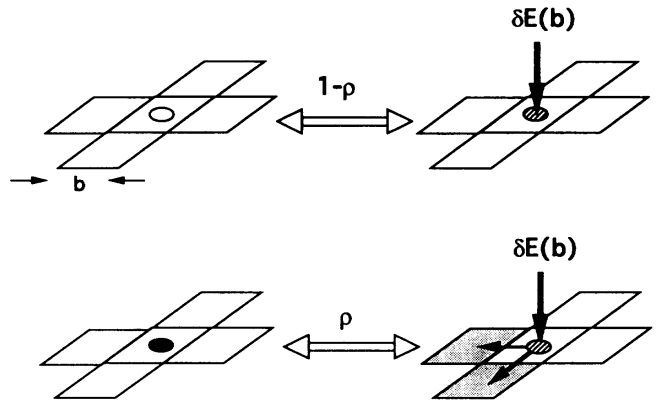

FIG. 3. Characterization of the static properties of coarse grained cells. A cell of generic scale $b$ is stable (white) if $\delta E(b)$ does not induce relaxation to the neighboring cells of the same size. In the opposite case the cell is unstable (black). The density of critical cells of scale $b$ is $\rho$.

formation and renormalization, these two vectors evolve to the same fixed point so that the two models belong to the same universality class.

The static properties for coarse grained variables are characterized as follows (Fig. 3): A cell of size $b$ is stable (white) if the addition of "quantum" of energy $\delta E(b)$, corresponding to scale $b$, does not induce relaxations in the neighboring cells. Of course internal relaxations can occur, but as long as they do not affect other cells we consider the cell stable. On the opposite the cell is critical (black) if the addition of $\delta E(b)$ induces relaxations into some neighboring cell(s). The parameter $\rho$ defines the density of the critical cells. The static and dynamical properties of the system are then fully characterized by the distribution $(\rho ; \bar{P})$.

We now proceed to define a renormalization transformation for the relaxation dynamics. Given a configuration $\alpha$ at a scale $b$ characterized by stable and critical subcells, we assume that one of the critical sites at scale $b / 2$ relaxes and study how energy is distributed to the neighboring cells of size $b$. An example of such a process is shown in Fig. 4. The index $\alpha$ that characterizes the configuration gives the number of critical subcells. In this case $\alpha=2$ and the weight of the configurations with two nearest critical subcells [Fig. 4(a)], considering that the energy distribution is uncorrelated [9], is simply

$$
W(\alpha=2)=4 \rho^{2}(1-\rho)^{2} .
$$

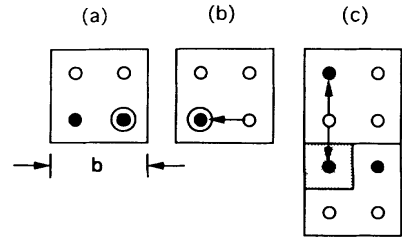

Microscopic relaxation processes (d)

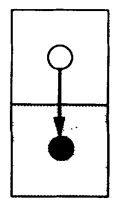

Coarse grained relaxation
FIG. 4. Example of the renormalization scheme for the relaxation dynamics. For details see the text. 
The process shown refers to the probability that the unstable subcell relaxes towards the other critical subcell [Fig. 4(b)]. This occurs with probability $(1 / 4) p_{1}^{(k)}$ where the index $(k)$ refers to relaxation processes at scale $b / 2$ and $(k+1)$ will refer to relaxation processes at scale $b$. At this point we consider the probability that the next relaxation event at scale $b / 2$ involves two neighboring sites of size $b / 2$, one inside and one outside the original cell of size $b$ [Fig. 4 (c)]. This occurs with probability $(2 / 3) p_{2}^{(k)}$. This series of relaxations at scale $b / 2$ contributes to the probability $p_{1}^{(k+1)}$ that characterizes the relaxation processes at scale $b$ [Fig. 4(d)].

By summing over all the processes that lead to $p_{1}^{(k+1)}$ one obtains for $\alpha=2$

$$
\begin{aligned}
p_{1}^{(k+1)}(\alpha=2)= & \left(\frac{1}{4} p_{1}^{(k)}+\frac{1}{6} p_{2}^{(k)}\right)\left(\frac{1}{2} p_{1}^{(k)}+\frac{2}{3} p_{2}^{(k)}+\frac{1}{2} p_{3}^{(k)}\right)+\left(\frac{1}{6} p_{2}^{(k)}+\frac{1}{4} p_{3}^{(k)}\right)\left(\frac{1}{2} p_{1}^{(k)}+\frac{1}{6} p_{2}^{(k)}\right) \\
& +\left(\frac{1}{6} p_{2}^{(k)}+\frac{1}{4} p_{3}^{(k)}\right)\left(\frac{3}{4} p_{1}^{(k)}+\frac{1}{2} p_{2}^{(k)}+\frac{1}{4} p_{3}^{(k)}\right) .
\end{aligned}
$$

In a similar way one can also write the expressions for $p_{2}^{(k+1)}, p_{3}^{(k+1)}$, and $p_{4}^{(k+1)}$. Finally, these probabilities are normalized for each configuration $\alpha$. This calculation should then be repeated also for the configurations $\alpha=3$ and 4. The configuration $\alpha=1$ is not included in the renormalization of the dynamics because it cannot lead to relaxation processes that span the whole cell of size $b$. The calculation is rather laborious but straightforward and it will be reported in detail in a long paper [16]

The renormalization of the relaxation processes alone would lead to trivial fixed points because the system simply decays. In order to describe the dynamics of the critical state it is necessary to couple the dynamics to a stationarity condition similar to the one used in Ref. [14] to define the average energy of the critical state. This means that, given a cell of size $b$, on average, the energy that goes in the cell should be equal to the energy that goes out. If we assume to inject a "quantum" of energy $\delta E(b)$, the probability that the cell relaxes is $\rho^{(k+1)}$ and, once this happens, it can occur with the four possible situations we have discussed previously (Fig. 2). This leads to the equilibrium condition

$$
\begin{aligned}
\delta E(b)=\rho^{(k+1)}[ & \delta E(b) p_{1}^{(k+1)}+2 \delta E(b) p_{2}^{(k+1)} \\
& \left.+3 \delta E(b) p_{3}^{(k+1)}+4 \delta E(b) p_{4}^{(k+1)}\right]
\end{aligned}
$$

that couples the dynamical properties to the static ones and provides a feedback mechanism that is an essential element for the process of self-organization [17] and for the definition of the nonlocal properties of the dynamics.

The structure of our renormalization scheme is therefore the following. Given $\left(\rho^{(k)} ; p_{n}^{(k)}\right)$ one defines the transformation $F_{n}$ for the dynamics at the scale $(k+1)$ for each configuration $\alpha$,

$$
p_{n}^{(k+1)}(\alpha)=F_{n}\left[\left\{p_{n^{\prime}}^{(k)}\right\}\right] \quad\left(n, n^{\prime}=1,4\right) .
$$

These probabilities are then averaged over the configurations $\{\alpha\}$ whose weight $W(\alpha)$ is defined by $\rho^{(k)}$,

$$
p_{n}^{(k+1)}=\left\langle p_{n}^{(k+1)}(\alpha)\right\rangle=\sum_{\alpha=1,3} W(\alpha) p_{n}^{(k+1)}(\alpha) .
$$

The equilibrium condition Eq. (4) provides then the renormalized density of critical sites at scale $(k+1)$, independent of the definition of $\delta E(b)$. The approximations involved in this scheme are due to the specific im- plementation of the spanning condition and to closure of the renormalization equations [16].

Given this scheme we can now start from a small scale state characterized by $\left(\rho^{(0)} ; \bar{P}^{(0)}\right)$ and study how this will evolve under scale transformation. If we start with a small scale dynamics characterized by the model of Manna $[8]$ we have $\bar{P}^{(0)} \equiv(0,1,0,0)$. Considering a low density of critical sites $\rho^{(0)}=0.1$, the evolution under scale transformation is shown in the upper part of Table I. In the lower part we show instead the evolution of the BTW [1] four-state model, starting from a large density $\rho^{(0)}$ $=0.9$.

Both models lead to the same asymptotic $(k \rightarrow \infty)$ fixed point dynamics $\left(\rho^{*} ; \bar{P}^{*}\right)$, so they belong to the same universality class. In addition we have checked that all models of this type also belong to this universality class.

TABLE I. Renormalization transformation for the static ( $\rho$ ) and dynamic $\left\{p_{n}\right\}$ properties for two sandpile models. The index $k$ refers to a change of scale. The limit $k \rightarrow \infty$ identifies the attractive fixed point $\left(\rho^{*} ; \bar{P}^{*}\right)$ that is the same for both models.

\begin{tabular}{cccccc}
\hline $\begin{array}{c}\text { Number of } \\
\text { iterations } \\
(k)\end{array}$ & $\rho$ & $p_{1}$ & $p_{2}$ & $p_{3}$ & $p_{4}$ \\
\hline \multicolumn{5}{c}{ Manna two-state model } \\
0 & 0.1 & 0 & 1 & 0 & 0 \\
1 & 0.612 & 0.436 & 0.495 & 0.068 & 0.001 \\
2 & 0.575 & 0.405 & 0.463 & 0.118 & 0.013 \\
3 & 0.542 & 0.362 & 0.456 & 0.158 & 0.024 \\
4 & 0.518 & 0.324 & 0.434 & 0.188 & 0.033 \\
$\infty$ & 0.468 & 0.240 & 0.442 & 0.261 & 0.057
\end{tabular}

BTW four-state model

\begin{tabular}{ccllcc}
0 & 0.9 & \multicolumn{1}{c}{0} & \multicolumn{1}{c}{0} & 0 & 1 \\
1 & 0.252 & 0 & 0 & 0.033 & 0.967 \\
2 & 0.308 & 0 & 0.012 & 0.726 & 0.262 \\
3 & 0.353 & 0.030 & 0.261 & 0.553 & 0.152 \\
4 & 0.388 & 0.090 & 0.357 & 0.437 & 0.116 \\
$\infty$ & 0.468 & 0.240 & 0.442 & 0.261 & 0.057 \\
\hline \hline
\end{tabular}


The fixed point is attractive and it allows us therefore to understand the self-organized nature of the critical stationary state.

The fixed point parameters $\left(\rho^{*} ; \bar{P}^{*}\right)$ provide a complete characterization of the static and dynamical behavior of the system at large scale and can be tested by suitable computer simulations. In addition one can derive analytically the critical exponents.

The avalanche exponent $\tau$ can be obtained as follows. The existence of a nontrivial fixed point with respect to the scale transformation guarantees that the properties of the system will be described by power law distributions. Therefore we can assume a power law avalanche distribution and relate the exponent $\tau$ to the fixed point properties $\left(\rho^{*} ; \bar{P}^{*}\right)$ shown in Table I. The first step is to transform the avalanche distribution in a distribution for the size $r$ of the clusters. This is related to the total number of sites $S$ by $S \simeq r^{D}$. It is easy to show with FST methods [18] that sandpile clusters in two dimensions are not fractal but compact and therefore $D=2$ in agreement with simulations $[1,6,7]$. The size distribution is therefore from $P(r) d r=r^{(1-2 \tau)} d r$. By using discrete length scales $b^{(k)}=2^{k} \times b_{0}$, we define the parameter $K$ as the probability that an active relaxation process is limited between the scales $b^{(k-1)}$ and $b^{(k)}$ and it does not extend further. This can be expressed as

$$
K=\int_{b^{(k-1)}}^{b^{(k)}} P(r) d r / \int_{b^{(k-1)}}^{\infty} P(r) d r=1-2^{2(1-\tau)} .
$$

Note that this is different from the quantity $\left(1-\rho^{(k)}\right)$ that includes the situation in which no relaxation occurs even at scale $b^{(k-1)}$. The parameter $K$ is defined by the condition that a relaxation event occurs at scale $b^{(k)}$ with the dynamics defined by $\left\{p_{n}{ }^{(k)}\right\} \quad(n=1,4)$, and that this process should stop and not affect the neighboring cells. Asymptotically $(k \rightarrow \infty)$ we can therefore express $K$ in terms of our fixed point parameters in the following way:

$$
\begin{aligned}
K= & p_{1}^{*}\left(1-\rho^{*}\right)+p_{2}^{*}\left(1-\rho^{*}\right)^{2}+p_{3}^{*}\left(1-\rho^{*}\right)^{3} \\
& +p_{4}^{*}\left(1-\rho^{*}\right)^{4}=0.296 .
\end{aligned}
$$

Using Eqs. (7) and (8) the exponent $\tau$ is then given by

$$
\tau=1-\frac{1}{2} \frac{\ln (1-K)}{\ln 2}=1.253 \text {. }
$$

This value of $\tau$ is in good agreement with the computer simulations on large systems that give $\tau=1.22$ [7] and $\tau=1.28[8]$.

It should be noted that the method we have used to compute the critical exponent $\tau$ is analogous to the FST approach to fractal growth [19] and it is related directly to the fixed point parameters. In usual critical phenomena exponents are derived instead from the derivative of the RG transformation with respect to the "critical" parameter. In SOC models this is impossible because the fixed point is attractive and the exponents that are related to the distance from the critical point do not exist. Along the same lines it is possible to compute also the dynamical exponent $z=1.234$, in good agreement with Refs. $[6,7,10]$. Details of these calculations will be reported in Ref. [16].

The introduction of a dissipation parameter $\gamma$ can also be considered in our renormalization scheme and this should be also renormalized in its turn. The main result is that dissipation introduces a length scale into the system and destroys the SOC properties [16]. In our scheme, in fact, the introduction of $\gamma$ turns the fixed point into a trivial one. This is in agreement with the simulation on large systems [11] of the type that we have discussed here. Instead, the dissipative model of Ref. [12] seems to belong to a different universality class.

It is a pleasure to thank P. Bak and D. Dhar for interesting discussions.

[1] P. Bak, C. Tang, and K. Wiesenfeld, Phys. Rev. Lett. 59, 381 (1987); Phys. Rev. A 38, 364 (1988); P. Bak and M. Creutz, in Fractals and Disordered Systems, edited by A. Bunde and S. Havlin (Springer-Verlag, Heidelberg, 1993), Vol. II.

[2] T. Vicsek, Fractal Growth Phenomena (World Scientific, Singapore, 1992).

[3] L. P. Kadanoff, Physica (Amsterdam) 163A, I (1990); see also Phys. Today 44, No. 3, 9 (1991).

[4] L. Pietronero, C. Erzan, and C. Evertsz, Phys. Rev. Lett. 61, 861 (1988). For a recent review, see A. Erzan, L. Pietronero, and A. Vespignani, "The Fixed Scale Transformation Approach to Fractal Growth" (to be published).

[5] L. P. Kadanoff, S. R. Nagel, L. Wu, and S. Zhou, Phys. Rev. A 39, 6524 (1989).

[6] S. S. Manna, Physica (Amsterdam) 179A, 249 (1991).

[7] S. S. Manna, J. Stat. Phys. 59, 509 (1990).

[8] S. S. Manna, J. Phys. A 24, L363 (1991).

[9] P. Grassberger and S. S. Manna, J. Phys. (Paris) 51, 1077 (1990).

[10] D. Dhar, Phys. Rev. Lett. 64, 1613 (1990); D. Dhar and R. Ramaswamy, Phys. Rev. Lett. 63, 1659 (1989); D. Dhar and S. N. Majumdar, J. Phys. A 23, 4333 (1990).

[1 1] S. S. Manna, L. B. Kiss, and J. Kertesz, J. Stat. Phys. 61, 923 (1990).

[12] Z. Olami, H. J. S. Feder, and K. Christensen, Phys. Rev. Lett. 68, 1244 (1992); K. Christensen and Z. Olami, Phys. Rev. A 46, 1829 (1992).

[13] Y. C. Zhang, Phys. Rev. Lett. 63, 470 (1989).

[14] L. Pietronero, P. Tartaglia, and Y. C. Zhang, Physica (Amsterdam) 173A, 22 (1991).

[15] P. Bak and K. Sneppen, Report No. BNL 49030 (to be published); M. Paczuski, S. Maslov, and P. Bak (to be published).

[16] A. Vespignani, S. Zapperi, and L. Pietronero (to be published).

[17] D. Sornette, J. Phys. I (France) 2, 2065 (1992).

[18] L. Pietronero and W. R. Schneider, Phys. Rev. Lett. 66, 2336 (1991).

[19] R. Cafiero, L. Pietronero, and A. Vespignani, Phys. Rev. Lett. 70, 3939 (1993). 


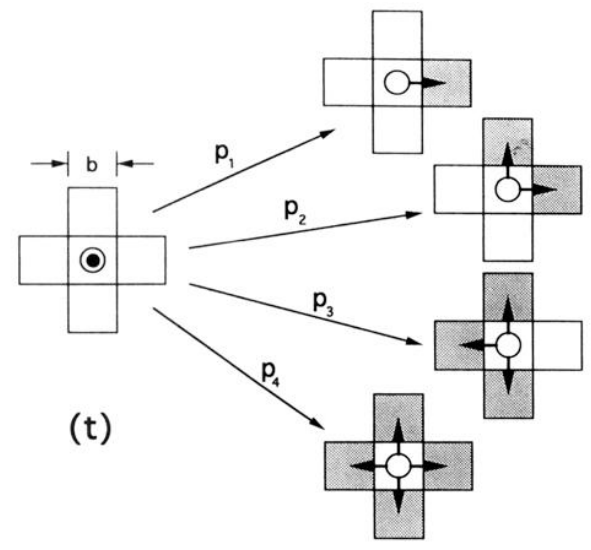

$(t+1)$

FIG. 2. No matter what is the small scale dynamics, at a generic scale $(b)$ relaxation can occur in the four possible ways shown. These four possibilities characterize the phase space of the dynamics of the system that will be used in the relaxation scheme. The probabilities refer to the number of neighboring cells influenced by the relaxation, independently of their position. 

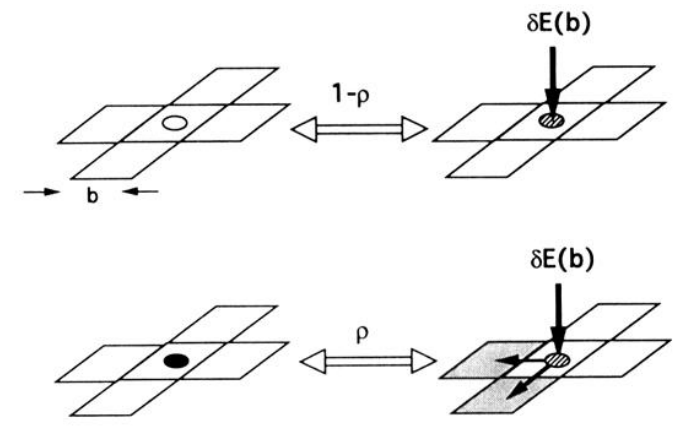

FIG. 3. Characterization of the static properties of coarse grained cells. A cell of generic scale $b$ is stable (white) if $\delta E(b)$ does not induce relaxation to the neighboring cells of the same size. In the opposite case the cell is unstable (black). The density of critical cells of scale $b$ is $\rho$. 


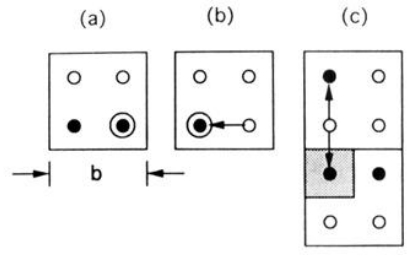

Microscopic relaxation processes (d)

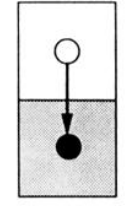

Coarse grained

relaxation

FIG. 4. Example of the renormalization scheme for the relaxation dynamics. For details see the text. 